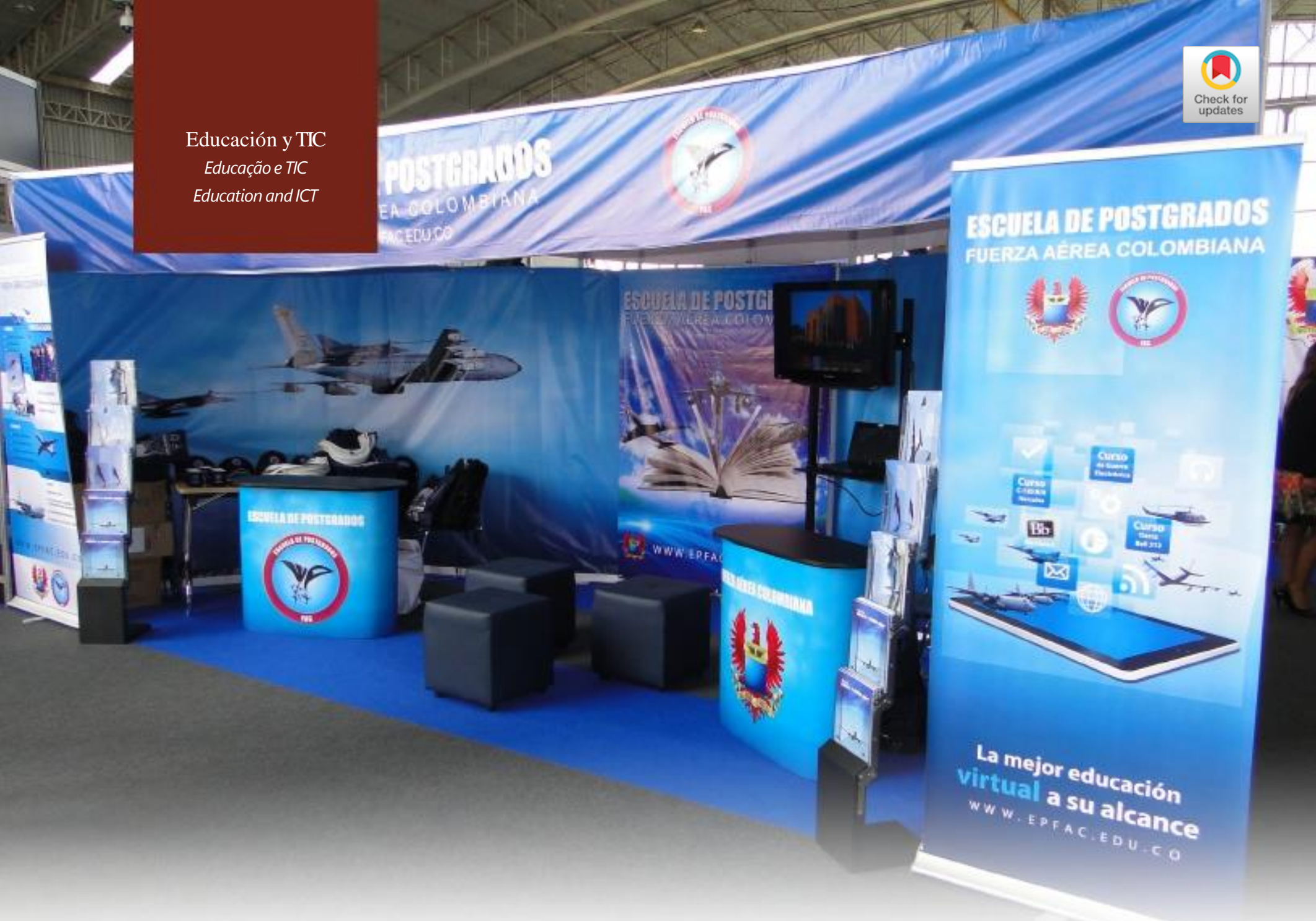

\title{
CódIGOS QR BASADO EN EL APRENDIZAJE MÓVIL COMO ESTRATEGIA PARA LA INVESTIGACIÓN FORMATIVA: UN CASO PILOTO ${ }^{1}$
}

CÓDIGOS QR BASEADOS NA APRENDIZAGEM MÓVEL COMO UMA ESTRATÉGIA DE PESQUISA FORMATIVA: UM CASO PILOTO² QR CODES BASED ON MOBILE LEARNING AS A STRATEGY FOR FORMATIVE RESEARCH: A PILOT CASE ${ }^{3}$

Erika Juliana Estrada-Villa ${ }^{4}$

Escuela de Postgrados de la Fuerza Aérea Colombiana

CIENCIA Y PODER AÉREO

ISSN 1909-7050 / E- ISSN 2389-2468 / Volumen 11/ Enero-diciembre de 2016/ Colombia/ Pp. 230-241

Recibido: 14/07/2016

Aprobado evaluador interno: 05/09/2016

Aprobado evaluador externo: 06/10/2016

Doi: http://dx.doi.org/10.18667/cienciaypoderaereo.500 
Para citar este artículo:

Estrada-Villa, E. J. (2016). Códigos QR basado en el aprendizaje móvil como estrategia para la investigación formativa: un caso piloto. Ciencia y Poder Aéreo, 17 (1), 230-241. Doi: http://dx.doi.org/10.18667/cienciaypoderaereo.500

1 Artículo de reflexión resultado de investigación del proyecto titulado: Estrategias de Formación en Investigación en Educación Superior Fase I, vinculado al Grupo de Investigación CIPAER.

${ }^{2}$ Artigo de reflexão resultado da pesquisa do projeto titulado: Estratégias de formação em pesquisa de educação Superior Fase I, vinculado ao Grupo de Investigação CIPAER.

${ }^{3}$ Reflection article as a result of the research project titled: Formation Strategies in Educational Research Phase I, linked to the Research group CIPAER.

4 Docente investigadora de la Especialización en Gerencia de la Seguridad Operacional / Maestría en Seguridad Operacional y tutora de trabajos de grados de la Escuela de Postgrados de la Fuerza Aérea Colombiana. Doctorado (en curso) en Educación y Sociedad, Msc. Informática Educativa y Esp. en Docencia Universitaria. Correo electrónico: erikajulianaestrada@hotmail.com
Resumen: este artículo brinda una estrategia con herramientas que conducen al interesado a plantear un problema de investigación a través de incorporar la tecnología móvil. Identificadas las necesidades, se diseñó un ambiente de aprendizaje mediado por dispositivos móviles con el fin de desarrollar habilidades para la identificación de un problema en el campo de la seguridad aérea a través de los códigos QR. La investigación se soporta en un estudio de caso con enfoque cualitativo; la obtención de los datos se realizó mediante la observación y el diseño de instrumentos, empleados para describir la dinámica de la integración de los dispositivos móviles en la formulación del problema de investigación; la muestra la conformaron catorce estudiantes de la Especialización en Gerencia de la Seguridad Operacional (EGSO), los resultados obtenidos se analizaron desde las categorías a priori: herramientas formulación del problema y colaboración, y las categorías emergentes: APP como recurso educativo yaccesibilidad. En suma, se presenta el análisis frente al diseño del problema de investigación con los códigos QR. Esta investigación se llevó a cabo en la (EGSO), con el propósito de contribuir a la búsqueda de estrategias efectivas para que los estudiantes decidan incorporar a sus proyectos de investigación.

Palabras clave: aprendizaje móvil; códigos QR; investigación formativa; problema de investigación.

Resumo: esse artigo fornece uma estratégia com ferramentas que levam ao interessado para plantear um problema de pesquisa através de introduzir a tecnologia móvel. Identificadas as necessidades foi desenhado um ambiente de aprendizagem mediante dispositivos móveis com a finalidade de desenvolver habilidades para a identificação dum problema no campo da segurança aérea através dos códigos QR. A pesquisa é suportada dentro dum estudo de caso focado no qualitativo; a obtenção dos dados foi feita mediante a observação e o desenho de instrumentos, utilizados para descrever a dinâmica da integração dos dispositivos móveis na formulação do problema da pesquisa; da amostra foi integrada por quatorze estudantes da mestria em Gerência da Segurança Operacional (EGSO), os resultados obtidos foram analisados desde as categorias previamente: Ferramentas, Formulação do problema e Colaboração e as categorias emergentes: APP como Recurso Educativo e Acessibilidade. Em resumo geral, apresenta a reflexão sobre o problema do trabalho de pesquisa com os códigos bidimensionais, e realizada esta investigação na Mestria em Gestão da Segurança operacional com o objetivo de ajudar para a busca de estratégias eficazes para os alunos decidir incorporar nos seus projetos de pesquisa.

Palavras-chave: aprendizagem móvel; códigos QR; pesquisa formativa; problema de pesquisa.

Abstract: This article provides a strategy with tools that lead the interested to pose a research problem through incorporating mobile technology. Having identified the needs, a learning environment mediated by mobile devices was designed in order to develop skills to identify a problem in the field of aviation security through QR codes. The research is supported by a case study with a qualitative approach; the data was obtained through observation and instrument design, used to describe the dynamics of the integration of mobile devices in the formulation of the research problem; the sample consisted of fourteen students of the Specialization in Operational Security Management (EGSO), the obtained results were analyzed from the initial categories: problem formulation and collaboration tools, and emerging categories: APP as an educational resource and accessibility. Overall, the analysis of the research problem design with QR codes is presented. This research was carried out in the (EGSO), with the purpose of contributing to the search for effective strategies for students to choose to incorporate into their research projects.

Key Words: Formative Research; Mobile Learning; Research Problem; QR Codes. 


\section{Introducción}

Actualmente son más las instituciones educativas que han ido incorporando constantemente el uso de las TIC en los procesos de enseñanza y aprendizaje, son diversas las razones que han llevado a esta masificación e implementación, en algunos casos se hace con el fin de ampliar las propuestas académicas, en otros para ampliar su cobertura o para integrar los aprendizajes en los programas académicos con el apoyo de la educación virtual.

De esta manera, los ambientes virtuales aumentan su popularidad e importancia, tanto entre los docentes, como en los estudiantes de educación superior, cambiando radicalmente el concepto de formación, donde el estudiante participa como actor principal y donde las TIC son un instrumento de apoyo al proceso de enseñanza-aprendizaje (Orrego \& Alba, 2013).

Es por esto, que los códigos QR (viene de sus siglas en inglés Quick Response) mediante el aprendizaje móvil, se convierten en una de tantas alternativas para enriquecer los ambientes de aprendizaje; que constituyen espacios donde las nuevas tecnologías como: geolocalización, internet, multimedia entre otros, se han potencializado rebasando el entorno académico tradicional; que a su vez favorece el conocimiento, la apropiación de contenidos, las experiencias y los procesos pedagógicos. Dichos ambientes de aprendizaje mediados por dispositivos móviles, se han expandido gracias a la inclusión del mobile learning ( $m$-learning) en los procesos de enseñanza-aprendizaje, fundamentado en el trabajo colaborativo, los conocimientos previos de los estudiantes y las ventajas que ofrece el aprendizaje ubicuo.

Teniendo en cuenta lo anterior, los códigos QR, fueron empleados como parte de una estrategia para la investigación formativa, porque se observó en la práctica educativa, que los estudiantes de la Especialización en Gerencia de la Seguridad Operacional (EGSO) presentaban dificultades para plantear el problema de investigación. Lo cual, se reflejaba en las estadísticas de no graduados del programa, con un $52.11 \%$ y una tasa de retención del $10 \%$ para once cohortes de la Especialización en Gerencia de la Seguridad Aérea (EGSA) (Secretaria General, 2014). Esto probablemente debido a que los estudiantes demostraron falta de interés frente a adelantar su opción de grado, lo cual podía deberse a unos inadecuados planteamientos del problema. Por otro lado, hay que tener en cuenta la pertinencia de la investigación en el programa de EGSO, la cual se configura cuando el problema que se ha planteado contribuye a solucionar un problema de la seguridad aérea en el sector aeronáutico institucional o del País. Por tanto, la pertinencia social de la investigación será entonces esta- blecida, sí muestra como la investigación puede responder a las preocupaciones del sector aeronáutico en general ( $\mathrm{Di}$ rectiva permanente 028,2013 ).

Es por esto, que el objetivo de esta investigación es incorporar la tecnología móvil, de manera que permita cautivar al estudiante, y lo conduzca por una ruta sistemática en su investigación, garantizado en la transferencia de conocimiento y del trabajo colaborativo. Es así, como se llegó a construir la unidad del módulo de investigación del programa EGSO de la Escuela de Postgrados de la Fuerza Aérea Colombiana (EPFAC) apoyada en los códigos QRy en la utilización de dispositivos móviles como herramienta de acceso a contenidos.

Para iniciar a comprender el tema, es necesario dar a conocer los conceptos sobre los cuales se fundamenta esta investigación: aprendizaje móvil y problema de investigación.

\section{Apuntes sobre aprendizaje Móvil o M-Learning}

Para Robles (2011) (citado en Estrada, 2014) el término aprendizaje móvil o $m$-learning ha surgido con el fin de asociar el uso de la tecnología móvil a el campo educativo. El aprendizaje móvil abarca muchas actividades que suceden fuera de una situación formal del aula de clase. Éste se refiere específicamente al aprendizaje que es facilitado por la utilización de herramientas móviles que pueden ser llevadas y utilizadas en cualquier momento y cualquier lugar, tales como: teléfonos celulares, reproductores de audio, tabletas y teléfonos celulares inteligentes entre otros. Asimismo, una idea de Sariola (2011) leída en la publicación de Robles (2011) indica que desde la perspectiva tecnológica existen tres características del aprendizaje móvil de las cuales la tercera puede beneficiar la perspectiva educacional:

1. portabilidad, se refiere a lo liviano de los equipos, fáciles de llevar y permiten movilidad,

2. inalámbrico, y

3. movilidad, se refiere a que las personas pueden tener acceso a la tecnología mientras se desplazan de un lado a otro.

Características como lo son la conveniencia y la relevancia, adquieren significancia dado que los estudiantes y docentes se pueden mover, y simultáneamente realizar o conducir a actividades educativas.

De acuerdo con lo anterior y extrapolando la información de Sariola \& Robles (2011), se puede decir que el 
aprendizaje móvil está en cualquier parte y en cualquier lugar, además consiste en añadir contenidos generados por herramientas basadas en tecnologías de la información y la comunicación (TIC) a un entorno del cual también se puede aprender. En concordancia, el $m$-learning más que una definición y/o herramienta tecnológica, se está convirtiendo en una nueva forma de ver lo que nos rodea; $y$ pues como lo propuso el Informe Horizon. El horizonte del aprendizaje móvil ya se encuentra entre nosotros y se puede decir que desde los inicios de los años 90 se ha venido investigando, logrando que hoy, haya una gran cantidad de experiencias de aprendizaje móvil en educación superior en Colombia y en el mundo, marcando una nueva tendencia.

Desde inicios de la educación, esta se ha apoyado en tecnologías que van desde un lápiz hasta lo que se ve en la actualidad como lo es la realidad aumentada, entre otras, buscando siempre favorecer el aprendizaje, una idea de esto lo expresa Facundo Ángel (2014) quien menciona en una entrevista que: "con tal de lograr el objetivo de la educación, que es ser y saber hacer para innovar, ya, utilicemos lo que esté a nuestro alcance, eso implica que la triada entre recursos o medios en este caso didáctica y pedagogía tiene que darse ya, el recurso esta y el recurso no es más que una herramienta, una muleta." (Estrada, 2014, p.112).

En asocio con el aprendizaje móvil, surgen otros conceptos, términos y teorías que lo refuerzan, como lo es el aprendizaje ubicuo, el cual permite recibir instrucción sin una fecha y lugar definido, y surge de la unión del aprendizaje en línea personalizado y del aprendizaje móvil; al combinar la enseñanza individualizada y la instrucción en cualquier lugar y a cualquier hora (Jones \& Jo, 2004). En concordancia, el conectivismo de Siemens \& Downes (2004), como teoría emergente del aprendizaje digital, lo explican como el aprendizaje (conocimiento explicito) puede residir fuera de nosotros (ej.: contenidos digitales) y está enfocado en conectar conjuntos de información especializada, y son esas conexiones las que permiten aprender más. Por último, se aborda el aprendizaje situado que tiene como característica dentro de los procesos educativos, y és que el aprendizaje está tanto fuera como dentro del aula de clase y éste a su vez avanza con la interacción social colaborativa (Soler, 2010).

Visto el aprendizaje móvil, se pasa a describir los códigos $Q R$, donde su objetivo básico es almacenar la máxima información en el mínimo espacio de forma visible y de fácil legibilidad.

\section{QR Code ${ }^{\circledR}:$ Quick Response Code}

El código QR es un sistema para almacenar información en una matriz de puntos o un código de barras bidimensional creado por Masahiro Hara para la compañía japonesa Denso-Wave en 1994 (Perez \& Lagos, 2014). Sus predecesores fueron los populares códigos de barras, muy comunes en los productos de los supermercados, los cuales aparecieron en 1977. Los códigos lineales (ver Figura 1a) de una dimensión almacenan información en una dirección y se deben leer en sentido vertical, mientras que los códigos $Q R$ (ver Figura 2b), almacenan información en ambas direcciones, y como su nombre lo indica son de respuesta rápida (Fernández Ordóñez, 2009).

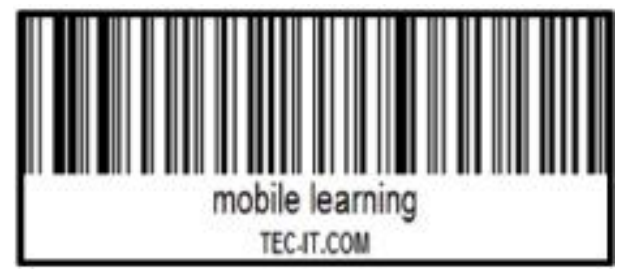

a) Codigo Líneal

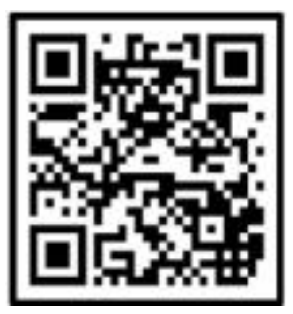

b) Código QR

Figura 1. Códigos lineales el origen de los códigos bidimensionales. Fuente: Tec.IT.com

Inicialmente los Códigos QR se utilizaron para el registro de partes de vehículos en Japón pero en la actualidad tiene muchas aplicaciones a nivel mundial, especialmente para usuarios con teléfonos móviles con cámara, debido a que contienen un decodificador incorporado lo que permite la lectura de este tipo de códigos (Luque, 2012).

Un código QR según Luque (2012) consiste en un conjunto de puntos oscuros ubicados, según la codificación, en un patrón cuadrado sobre un fondo blanco que graba caracteres en dirección vertical y horizontal, de ahí su característica de 2D (Bidimensional) (ver Figura 2), que se puede utilizar mediante impresión o por pantalla para ser decodificado de forma sencilla y a alta velocidad desde lectores hardware o aplicaciones de software; y es superior a los códigos de barras porque permite almacenar hasta 7.089 caracteres numéricos, adicional a la capacidad de corregir errores y restaurar hasta un 30\% de los datos. 


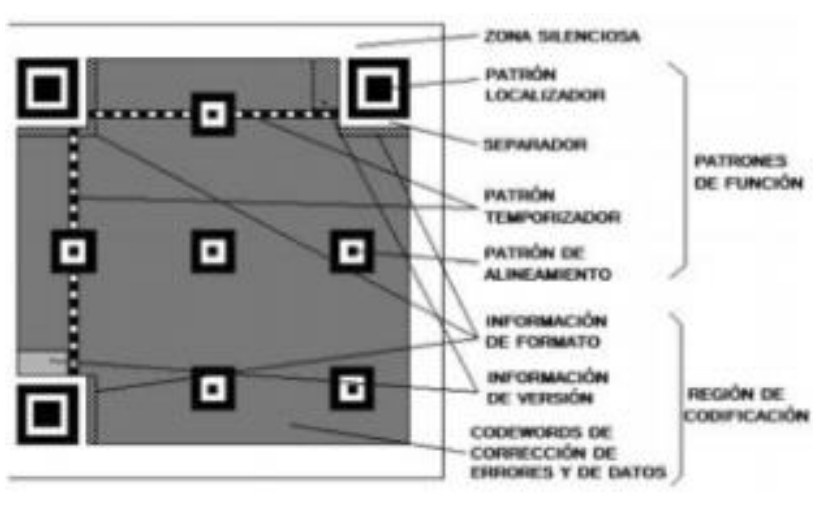

Figura 2. Estructura de un código QR

Fuente: Luque (2012, p.11).

Este tipo de códigos no sólo se han empleado en la automatización industrial, la publicidad o la gestión de inventarios, también esta tecnología ha arribado a la educación, debido al amplio acceso que se tiene de dispositivos móviles con cámara, unido a la fácil descarga de la aplicación "lector de códigos", escaneo y decodificación de la información incorporada de los códigos QR, lo que posibilita trabajar contenidos y dinamizar las actividades académicas.

De este modo, se hace factible trabajar la realidad aumentada en educación por intermedio de los códigos $Q R$, al agregar a un objeto físico capas de información digital. Entiéndase la realidad aumentada como el conjunto de recursos que permiten al usuario, en tiempo real, recibir e interactuar con información no percibida por sus sentidos sino a través de dispositivos externos de captura de la realidad.

\section{Apuntes sobre el problema de investigación}

La investigación en los programas de postgrado en la educación superior en Colombia la rige lo preceptuado en el decreto 1295 (MEN, 2010) y la define como las actividades que permiten desarrollar una actitud crítica y una capacidad creativa para encontrar alternativas para el avance de la ciencia, la tecnología, las artes o las humanidades, así como el espacio adecuado para encontrar soluciones a problemas sentidos de la Fuerza Aérea Colombiana (FAC) y del sector aeronáutico en general. A su vez, la investigación formativa es un conjunto de actividades que se realizan para familiarizar al estudiante con el proceso mismo de investigación y para formarlo como futuro investigador (Consejo Nacional de Acreditación , 2010).

Para los programas de postgrados la EPFAC, se determinó que un proyecto de investigación es aquel que se refiere a un conjunto articulado y coherente de actividades orientadas a alcanzar uno o varios objetos relacionados con la generación, adaptación o aplicación creativa de conocimiento.
Para ello, se sigue una metodología definida que prevé al logro de determinados resultados, bajo condiciones de recursos y tiempos especificados (Colciencias, 2014).

Lo anterior, se definió porque los estudiantes de la EGSO deben adelantar una opción de grado como requisito para optar el título de Especialistas (Reglamento de Investigación -EPFAC, 2012). Es por esto, que cuando los estudiantes ingresan al programa deben plantear un problema de investigación, y según lo dicho en la introducción no les resulta motivante.

Por otro lado, en una investigación después de haber definido y revisado el tema, el paso a seguir es plantear el problema de investigación, Johnson \& Christensen (2014) plantean que un problema de investigación está relacionado con un tema específico de un área del conocimiento el cual es susceptible de ser investigado.

El problema de investigación es fundamental, por lo general se formula con una pregunta específica, y es la que da la razón para la búsqueda de conocimientos, y a su vez debe estar delimitado en un marco temporal y espacial. Cabe aclarar que existen preguntas de investigación tanto para el enfoque cualitativo como cuantitativo, en las preguntas cuantitativas se relacionan y mencionan las variables. Las preguntas cualitativas no son específicas y se usan por lo general para explorar y entender un proceso o un fenómeno en particular (Bautista, 2011).

Por lo tanto, la pregunta de investigación cualitativa es alrededor de procesos y fenómenos explorados que buscan ser descritos, poseen la característica de marcar la ruta en la investigación. Esto puede ayudar a conseguir el propósito del estudio a través de resolver los componentes de las preguntas. La adecuada delimitación y formulación del problema de investigación, hace que el investigador reflexione constantemente sobre cada acción relacionada con su investigación puesto que los resultados deben estar ligados al problema de investigación.

Extrapolando lo anterior, un problema de investigación en la EGSO resulta pertinente sí soluciona un problema de la Fuerza Aérea Colombiana (FAC), que a su vez sea de interés del estudiante. Es decir, dentro de la EGSO la elección de un tema de investigación no puede escapar de la influencia de los valores y la misión institucional.

\section{Estudio de caso}

Lo que se presenta en adelante hace parte de un estudio de caso realizado por la investigadora, con la unidad de estudio del módulo de Investigación del programa EGSO 
ofrecido en la modalidad presencial y apoyado en la virtualidad. A continuación, se presentan las principales características identificadas en el estudio.

\section{Descripción: unidad observable del estudio de caso}

El programa de investigación se desarrolló bajo la modalidad blended learning mejor conocido como $b$-learning. Esta modalidad de aprendizaje se caracteriza por mezclar actividades de formación presencial con un momento del aprendizaje que se da en la virtualidad "Which combines face to face and virtual teaching" Coaten \& Marsh (2003) (citado en Bolivar, Ochoa \& Orrego, 2014). La presencialidad se dió en el aula durante cuatro semanas, y a su vez este estaba planeado como un espacio para el aprendizaje activo, dado que como lo plantea Huber (2008) "No es posible aprender por otra persona, cada persona tiene que aprender por sí misma" (p.65). Se propició el espacio para que los estudiantes realizaran construcciones colectivas de conocimiento con base en los planteamientos de las lecturas propuestas y sus conocimientos previos (Estrada, Rodríguez, Gutierrez \& Jaramillo, 2015). Los encuentros académicos combinaron presentaciones por parte de la docente así como discusiones, trabajo en grupo, exposiciones y aprovechando las ventajas del aprendizaje situado se realizó la actividad con los Códigos QR, facilitado por el contexto que ofreció oportunidades para aplicar conocimientos a través de la vinculación de los dispositivos móviles (Jonassen, Mayes \& McAleese, 1993 citado en Huber, 2008). El trabajo virtual fue a través de la plataforma Blackboard durante la fase de trabajo independiente con un ambiente virtual de aprendizaje diseñado por la docente, se aclara que los datos de esta fase no se incluyeron dentro de este artículo.

El objetivo de la actividad fue desarrollar habilidades para la identificación de un problema en el campo de la seguridad aérea a través de los códigos QR; y la competencia específica consistía en la"solución de problemas: observar, identificar, descubrir deficiencias en distintas situaciones y analizarlos crítica y sistemáticamente para definir alternativas e implementar soluciones."(Documento Maestro EGSO, p. 62, SNIES 102769, agosto de 2013).

\section{Actividad ancadémica Códigos QR}

La actividad con los códigos fue diseñada con el objetivo de brindar a los estudiantes la posibilidad de acceder a través de los dispositivos móviles y mediante los códigos QR a una estrategia que les facilitara plantear el problema de investigación (ver Figura 3), para lo cual se apoyó en contenidos publicados para la sesión en el ambiente virtual de aprendizaje implementado a través de la plataforma Blackboard.
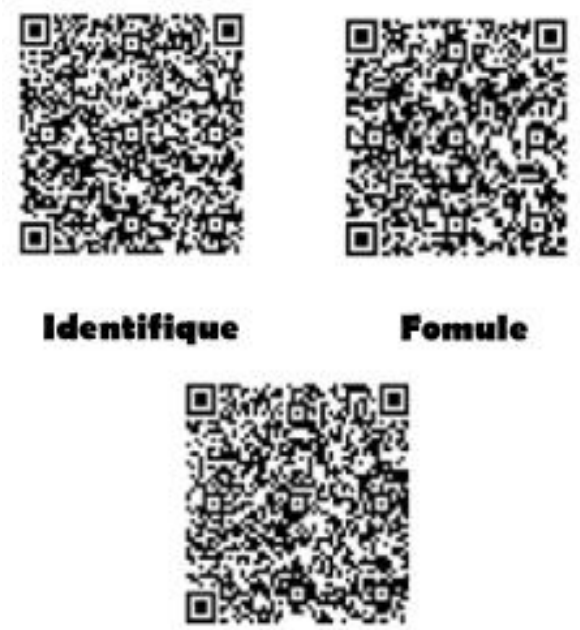

Cree

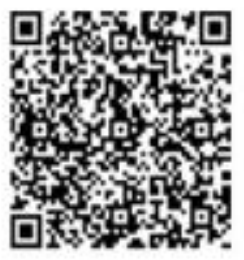

Aplique

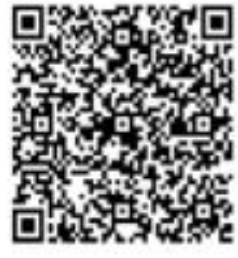

Comparta
Figura 3. Códigos QR empleados para trabajar el problema de investigación.

Fuente: elaboración propia.

Nota: descargue el APP que lee los códigos QR y escanee para acceder a la información.

Tabla 1.

Matriz para el planteamiento del problema dentro de la actividad de los códigos QR

\begin{tabular}{l}
\hline \multicolumn{1}{c}{ Aspectos identificables } \\
\hline Tema de interés \\
¿De dónde se origina? ¿Por qué? \\
Contexto \\
¿En dónde se ubica la situación problemá- \\
tica? ¿Quiénes están involucrados? Si es en \\
lo teórico ¿Qué concepciones se manejan? \\
Antecedentes \\
¿Qué se conoce sobre el tema? \\
Conceptos clave \\
¿Cuáles conceptos se deben definir? \\
Situación problemática concreta \\
Definición clara del problema \\
Posibles preguntas de investigación \\
Justificación del problema \\
¿A quién afecta esta idea de investigación? \\
¿Quién o qué se beneficia? ¿Cuáles son las \\
posibles contribuciones?
\end{tabular}


Los códigos permitieron a los estudiantes consultar la información, acceder al foro y al material para diligenciar la matríz para el planteamiento del problema (ver Tabla 1). Así como trabajar con la herramienta de compilación y análisis de problemas Flipchart o post-it, que a continuación se describe.

\section{Flipchart utilizando POST-I ${ }^{\circledR}$}

Pérez (2012) propone que en un"post-it ${ }^{\circledR}$ "cada integrante del equipo escriba una idea por papelito, en este caso respecto a su problema de investigación. Después estos se pegan en una superficie donde los estudiantes lo puedan observary con los elementos vistos en clase respecto al problema de investigación basados en Sampieri et al., (2010); puedan discutir, reunir las ideas pegándolas una encima de otra y esto permite analizarlas y organizarlas de acuerdo a los conocimientos previos (Ausubel, Novak\& Hanesian, 1983 citado en Miras, 2002). La forma como se visualiza la representación gráfica del flipchart tanto en la práctica como en lo teórico se presenta en la Figura $4 a$ y 4 b respectivamente.

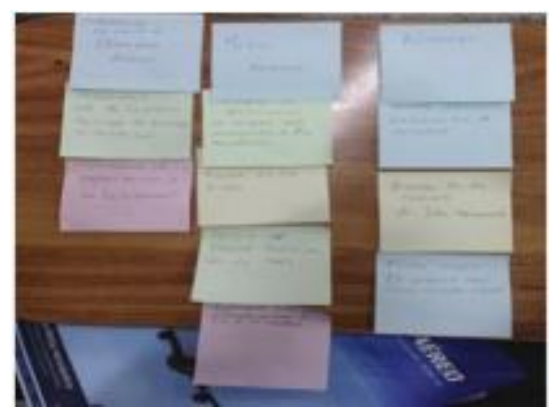

a) Actividad práctica Flipchart del problema a investigar.

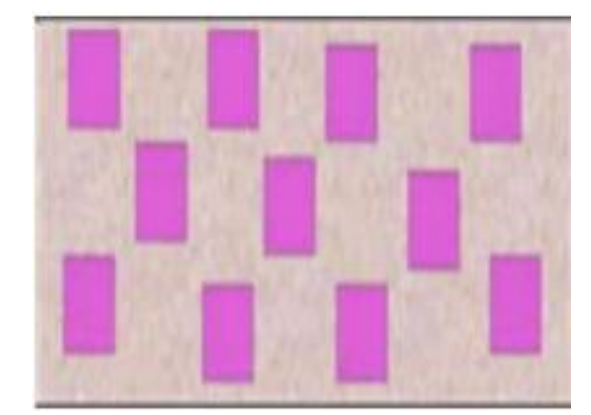

b) Metodología Flipchart por Perez-Uribe (2012, p.12).

Figura 4. Representación forma Flipchart.

Fuente: tomado y adaptado de Pérez-Uribe (2012, p.12).

\section{Diseño metodológico}

Esta investigación se enmarca en el estudio de caso y se fundamentó en lo dicho por Martínez- Carazo (2006) citando Eisenhardt (1989) quien lo concibe como "una estrategia de investigación dirigida a comprender las dinámicas presentes en contextos singulares" (p.174), lo cual se com- plementa con un enfoque cualitativo (Hernandez-Sampieri et al., 2010) dado que lo realizado en términos del diseño de la investigación busca describir la dinámica de la integración de los dispositivos móviles a través de los códigos $\mathrm{QR}$, como estrategia para la formulación del problema de investigación en catorce estudiantes de la Especialización en Seguridad Operacional Cohorte I de la Escuela de Postgrados de la Fuerza Aérea Colombiana; para posterior evaluación (Stake, 1995 citado en Johnson, B., \& Christensen, 2014) con el fin de comprender lo que está sucediendo en el proceso de enseñanza- aprendizaje combinando con distintos instrumentos para la recolección de los datos y para su posterior análisis por categorías a priori y emergentes dentro de la investigación.

\section{Obtención y procesamiento de los datos}

Para recoger los datos necesarios se evaluó la sesión para lo cual se diseñó un cuestionario que permitió tener información acerca de las actividades realizadas; lo anterior complementado con observaciones. La información de los instrumentos se detalla en el siguiente aparte.

\section{Análisis de los resultados}

Por otro lado, un objetivo de la investigación era indagar, describir la incorporación de los dispositivos móviles a las actividades académicas, entendidas estas como las actividades que el profesor utiliza para el desarrollo de su clase y que están orientadas por unos lineamientos curriculares y que en este caso tuvo el propósito de la investigación formativa.

Para este estudio, además de los datos descriptivos, se consideraron las siguientes categorías de análisis que se observan en la Figura 5.

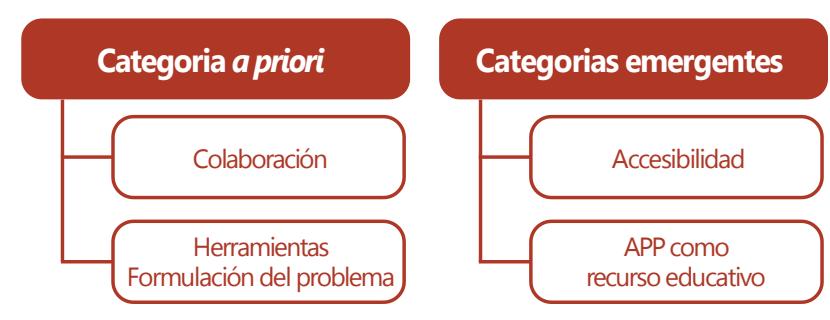

Figura 5. Categorías a priori vs categorías emergentes de la investigación.

Fuente: elaboración propia.

Las categorías a priori son analizadas en los instrumentos usados para la recolección de la información antes y después de la implementación del ambiente de aprendizaje en las jornadas de la sesión. 
Con el fin de determinar la vinculación de los códigos $\mathrm{QR}$, para apoyar los procesos de investigación formativa de la EGSO. El primer instrumento fue una rejilla denominada "matriz activación conocimientos previos" que se aplicó 14 estudiantes del 15 al 28 de marzo de 2014, la cual buscaba identificar la situación inicial de los estudiantes entorno a su investigación. Con el segundo instrumento se pretendía evaluar la actividad de integración de los códigos $Q R$ al problema de investigación, esta se aplicó durante el mes de mayo de 2014. Durante la implementación del ambiente de virtual de aprendizaje se hizo registro del desarrollo de cada uno de los planteamientos de los anteproyectos de investigación y de las concepciones acerca de los estudiantes acerca de las herramientas de trabajo colaborativo implementado a través del curso denominado opción de grado - Gerencia Seguridad Operacional 2014, que se impartió a través del Ambiente Virtual de Aprendizaje de la Fuerza Pública (AVAFP), que se soporta en la plataforma LMS BlackBoard.

\section{Instrumento 1. Rejilla "Matriz activación conocimientos previos"}

Los datos recogidos a través del instrumento arrojaron los siguientes resultados:

Herramientas formulación del problema. El 63,3\% de los estudiantes considera que el problema de investigación tiene algún significado con su profesión o el que hacer al interior de la FAC, el 16,7\% sugiere o tienen la intención de trabajar sobre un problema que le sea dado y el $19 \%$ sugiere que le hacen falta elementos para definir adecuadamente su problema de investigación.

De acuerdo al objetivo de la investigación sólo el $24 \%$ explicó el objetivo de la investigación. El 32\% elaboró un objetivo de la investigación y el 33\% realizó un objetivo laboral.

Referente al planteamiento del problema desde precedentes, experiencias y teorías, los estudiantes plantearon en un $50 \%$ documentos institucionales, un $26 \%$ plantearon sus propias experiencias y un $24 \%$ no conoce la información solicitada al respecto, siendo general el llamado a utilizar herramientas que les ayude en este aspecto.

Con relación al uso de los dispositivos móviles y las APP, se realizó un sondeo, donde los estudiantes afirmaron en un $50 \%$ preferir usar el celular para realizar las actividades dejadas en clase y se encontró que el $13 \%$ lo usa para investigación etiquetar y guardar los contenidos relacionados con su investigación y un 37\% lo usa para actividades laborales y administrativas.
Instrumento 2. Cuestionario evaluación actividad

\section{Códigos QR}

Se elaboró con el fin de conocer la percepción de los estudiantes en torno al proceso de enseñanza aprendizaje que se orientó. El cuestionario de evaluación arrojo lo siguiente: en la Figura 6, se observa que con respecto al uso de los códigos QR para apoyar la formulación del problema de investigación, un $50 \%$ calificó la actividad de excelente y el otro $50 \%$ de muy bueno. Se infiere que esto fue debido a la alta motivación que se evidenció en los estudiantes, y que las actividades propuestas generaron un reto que los llevó a plantear sus problemas de investigación.

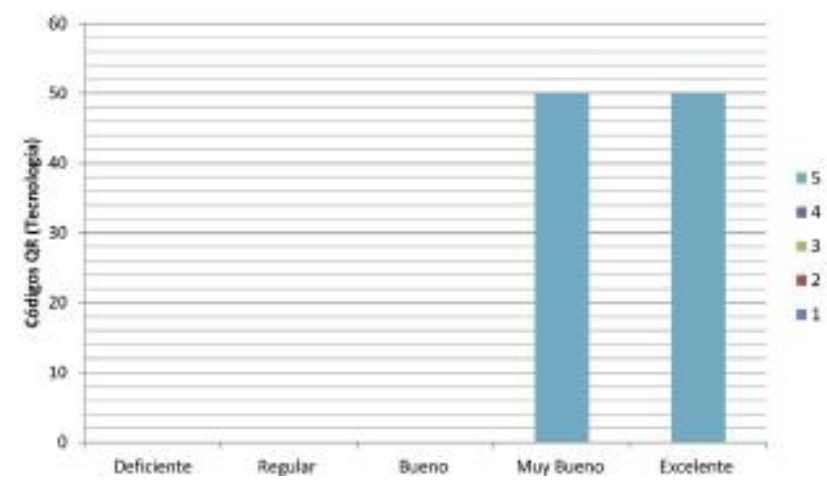

Figura 6. Los códigos bidimensionales (tecnologías).

Fuente: elaboración propia.

Nota. Los códigos bidimensionales (tecnologías) usadas en la sesión se alinean estrechamente con los objetivos de la sesión formulación del Problema de investigación.

Asimismo, la Figura 7 indagó acerca de sí los tiempos y las sesiones empleados en la actividad fueron los adecuados, a lo cual un $44.4 \%$ contesto que fue excelente, otro $44.4 \%$ contesto que fue muy bueno y el $11.1 \%$ mencionó que fue bueno. Respecto a la variable tiempo, se observó que hubo retrasado en el cronograma planteado para el desarrollo de la actividad, esto debido a los problemas de conectividad.

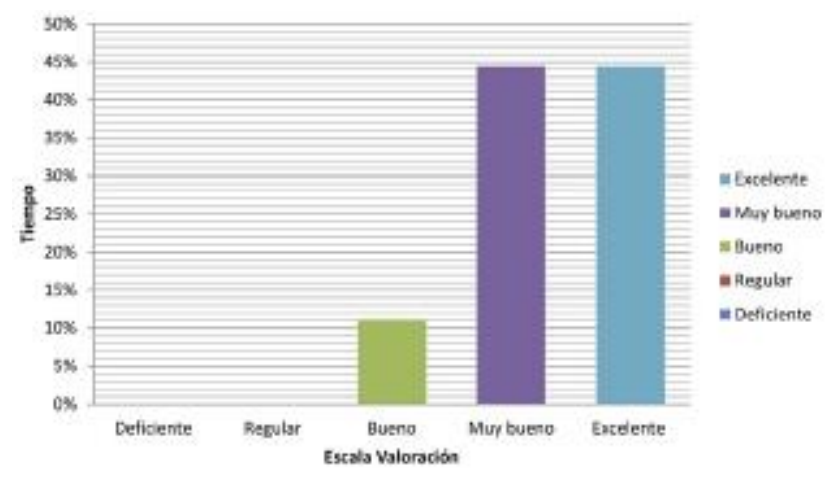

Figura 7. Tiempo en la sesión de la actividad de los códigos QR. Fuente: elaboración propia. 
Por otra parte, se indagó en sí los contenidos empleados en la actividad con los códigos QR contribuyó a la formulación del problema (ver Figura 8) a lo que en un $88.9 \%$ la califico de excelente y un $11.1 \%$ de muy bueno. Por otra parte, la gráfica relaciona otras variables, donde un $77.8 \%$ reconoce la importancia de los dispositivos móviles en los procesos académicos de la EGSO. Evidenciado en que en $66.7 \%$ y $33.33 \%$, es excelente y bueno respectivamente; de lo cual se infiere que el curso brindó los fundamentos teóricos y las herramientas para que se formularan adecuados problemas de investigación, y en respuesta a esto el $77.8 \%$ calificó de excelentes las orientaciones dadas dentro de la actividad para la formulación del problema de investigación.

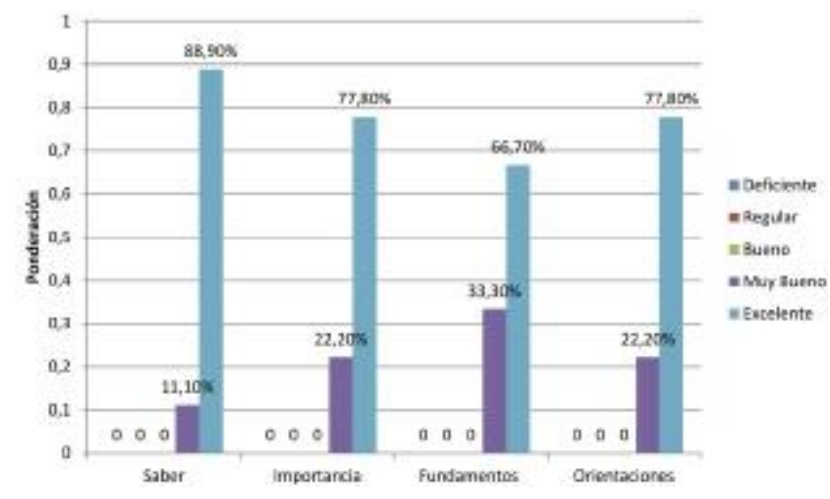

Figura 8. Percepciones respecto a las actividades con Códigos QR. Fuente: elaboración propia.

Nota. Respecto a los saberes, importancia, fundamentos y orientaciones percibidas por la muestra en las actividades con Códigos QR para el planteamiento del problema.

Los segmentos reducidos obtenidos del cuestionario aplicado a los estudiantes para determinar la facilidad y pertinencia de los códigos $Q R$, se presentan a continuación:

Sa: "Considero propicia la utilización de los códigos bidimensionales en la clase de metodología de investigación ya que nos motivó y nos ayudó a plantear la investigación"

Sj:"Me resultó fácil y dinámica la lectura de los códigos para el acceso a los contenidos."

Sd: "La consulta de los códigos a través de la blackboard brinda la posibilidad de acceder a la información de forma externa facilitando el auto aprendizaje"

Sc:"El acceso a los contenidos de investigación mediante los dispositivos móviles motiva a la consulta, cosa que no ocurre en las clases tradicionales"

Si: "El uso de los dispositivos móviles y las aplicaciones fue muy interesante el proceso de consultas para la formulación del proyecto de investigación"

\section{Observaciones de campo}

Las observaciones realizadas durante la implementación del ambiente de aprendizaje y las evidencias de la integración de los dispositivos móviles recogidas, presentan adicionalmente herramientas de trabajo colaborativo y una reflexión en la integración pedagógica de los dispositivos móviles a la práctica educativa. El siguiente análisis se organiza de acuerdo a las categorías: accesibilidad, APP como recurso educativo, colaboración e integración de dispositivos móviles.

Accesibilidad. Esta categoría surge del análisis de las dificultades que tuvieron los estudiantes respecto con el acceso a internet. Durante la implementación de la actividad se presentaron inconvenientes relacionados con la infraestructura (Estrada, 2014). Estas dificultades fueron registradas por el investigador (denominado iE) así:

iE: "(Sesión 1) Al iniciar la actividad se observa que los estudiantes tienen problemas para bajar la aplicación que lee los códigos, esto es debido a los problemas con el wifi de las aulas de la UMNG, sin embargo los estudiantes que cuentan con su plan de datos no presenta este inconveniente".

iE: "(Sesión 2 evidencias de la actividad) Se nota la demora en la velocidad de internet para el envió por correo electrónico del registro fotográfico de la actividad".

iE: "(Sesión 3. Creación Código QR) Se observa demora en la búsqueda en internet, así como se cae la página cuando se están generando el código".

iE: "(Sesión 4. Elaboración matriz planteamiento problema). Se observa lentitud a la hora de descargar la plantilla de la blackboard".

Otro inconveniente con la accesibilidad estuvo relacionado con las aplicaciones (APP) para llevar a cabo la actividad y esto fue dado al desconocimiento de la herramienta.

iE:"Los estudiantes que no contaban con la cuenta de correo en sus teléfonos, debieron bajarla y esto genero retrasos en la actividad, así como los que debieron bajar la aplicación de lectura de códigos y la APP Blackboard".

iE: "(Correo institucional para compartir) El envío de las evidencias de la actividad a la cuenta de correo institucional de la docente, reboto en la mayoría de la ocasiones".

Las observaciones del investigador dejan evidente la preocupación de algunos estudiantes por las fallas y demora en el internet: 
iE: "La mayoría de los estudiantes demostraron preocupación por no tener acceso fácil rápido a las herramientas para el desarrollo de la actividad ".

APP como recurso educativo. Las aplicaciones (APP) fueron un recurso fundamental para la implementación de las actividades tanto de los Códigos QR como del ambiente de aprendizaje. Las anotaciones del investigador ponen en evidencia este hecho:

iE: "Previamente se enviaron correos con instrucciones de la actividad".

iE: "La lectura de códigos bidimensionales se da a partir de las APP utilizadas para tal fin".

iE: "Se solicita a los estudiantes que utilicen la blackboard, enviar correos, trabajar la matriz, tomar fotos desde las aplicaciones de sus teléfonos móviles".

iE: "Se imprimieron los Códigos QR, se dispusieron en el aula pero a su vez se encontraban alojados en el espacio virtual del AVA y los estudiantes debían seguir la ruta allí indicada, así mismo se les pedía generar un código QR con la formulación de sus problemas de investigación".

Colaboración. Durante todo el proceso pedagógico se evidenció la colaboración entre los estudiantes y entre los grupos, algunas observaciones del investigador son:

iE:"... (Metodología post-it) es una herramienta creada para compartir conocimiento y experiencias para el planteamiento del problema de investigación".

iE: "...(en el AVA de la blackboard y en un código QR se encontraba el enlace compartir y participar en el foro relacionado con el código generado y lo realizado en la sesión".

iE: "Los estudiantes lograron hacer el código QR y lo comparten con los compañeros...entre grupos se comparten información y ayudas a través del whatsApp mientras trabajan en la actividad de la matriz".

iE: "Para esta actividad no se dieron instrucciones, estaban en los códigos, cada estudiante explora los códigos, los que van descubriendo explican a los otros como acceder a la información, comparten los datos de sus móviles y todos colaboran entre sín

La colaboración entre los estudiantes se hizo evidente en el trabajo de cada sesión pues los estudiantes comparten lo descubierto, reciben comentarios, preguntas y colaboran entre compañeros para alcanzar el objetivo de enseñanza.

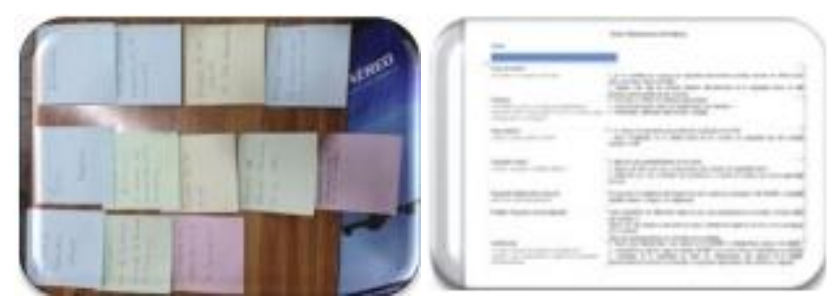

Trabajo colaborativo Post-it

Matriz planteamiento del problema

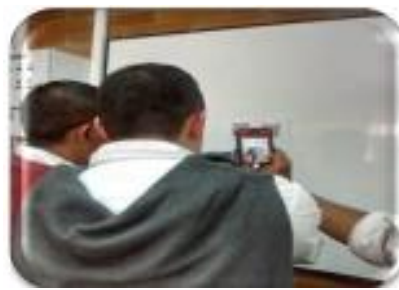

Trabajo colaborativo

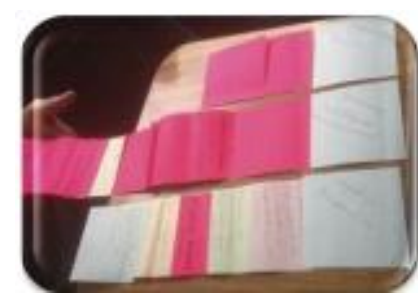

Matriz priorización de problemas

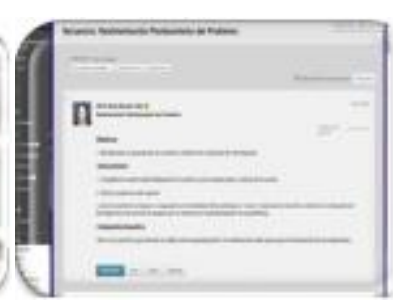

Compartiendo Matriz en el foro

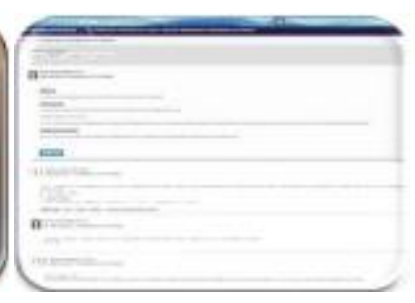

Foro
Figura 9. Evidencias del trabajo colaborativo durante el desarrollo de la investigación.

Fuente: elaboración propia.

\section{Reflexión final: integración de los dispositivos móviles}

Las herramientas usadas fueron un medio para desarrollar y alcanzar los objetivos propuestos. La sesión propició un espacio de reflexión y de trabajo colaborativo a través de la interacción con los dispositivos móviles.

La reflexión sobre trabajar el problema de investigación con el aprendizaje móvil facilitó delimitar y plantear el problema; y potencia la importancia de planear estrategias junto con el uso de las APP, la interacción y colaboración. Se encontró y se destacan algunos elementos a tener en cuenta para la integración de los dispositivos móviles en las actividades académicas, y son: la selección de los contenidos a trabajar, no es conveniente usar contenidos robustos por medio de las aplicaciones móviles. El diseño de estrategias de aprendizaje móvil debe llevar a los estudiantes, paso a paso a través de los objetivos planteados, el tema y las habilidades, al trabajo con los compañeros, así como la actividad debe ser supervisada y motivada por el docente. En cuanto a las herramientas empleadas, el docente debe tener conocimiento y experticia en el manejo de estas. Por último, la actividad que se diseñe debe ser innovadora, es- 
tar estructurada conceptualmente, y que a su vez pueda ser evaluada, para que así pueda ser replicada en otro entorno académico.

\section{Conclusiones}

La estrategia para presentar contenidos asociados a la investigación formativa en EGSO utilizando el aprendizaje móvil contribuyó de forma positiva en el planteamiento del problema de investigación y por ende aportó a que disminuyeran las tasas de retención del programa en relación con otras cohortes, dado que para el 2014 nueve de once estudiantes matriculados completaron sus créditos en los dos períodos académicos programados (Estadísticas SEGEN, 2015).

El uso de los códigos QR resulta económico debido a que el software requerido en los procesos de desarrollo, codificadores y decodificadores de los códigos generalmente es libre. Además, los dispositivos móviles permiten descargar software de códigos QR desde las tiendas de aplicaciones. Por otro lado, se observó que existe un factor limitante en el uso de los dispositivos móviles en las actividades académicas; y es la infraestructura, como lo plantea Estrada (2014), la conexión inalámbrica que debe ofrecer amplia cobertura y excelente capacidad, especialmente porque el uso de realidad aumentada requiere manejo de muchos recursos multimedia.

Desde las concepciones pedagógicas se concluye que la estrategia de investigación formativa implementada a través de la integración de los dispositivos móviles se fundamentó en el aprendizaje ubicuo, dada la combinación del aprendizaje en línea personalizado con el aprendizaje móvil. El objetivo del ambiente de aprendizaje se logró gracias al aprendizaje colaborativo, el cual se abordó continuamente dentro de las actividades académicas, donde los estudiantes además de estar adquiriendo información, indispensable para el planteamiento del problema de investigación, requirieron clasificar información y realizar distinciones al consumir los contenidos ofrecidos a través de los códigos QR y conectar con el medio de acuerdo a sus conocimientos. Las ventajas del trabajo colaborativo para el aprendizaje, Según González, M:; Muñoz, P.\& Hernández, N. (2014) (citando a Johnson, Johnson \& Holubec, 1993; Roberts, 2005; Slavin, 1985) están ampliamente recogidas en multitud de estudios. Asimismo, los autores afirman "que el trabajo colaborativo es una de las presencias dominantes en la formación apoyada en tecnologías y de ahí la importancia de las prácticas que se están desarrollando" (p.26). También, la investigación recoge elementos del aprendizaje situado, Brown \& Duguid (citado en Soler, 2006) lo definen como una nueva perspectiva acerca del proceso de aprendizaje, donde cualquier forma de aprendizaje, real o simulado, es necesariamente "Situado", donde el contexto o cultura incide sobre el tipo de aprendizaje que se realiza o se fomenta en la comunidad, y aclaran que este no constituye una nueva teoría o modelo instruccional. El estudio de caso buscó conocer la interacción entre el aprendizaje y las situaciones o contextos con dispositivos móviles en una práctica educativa en educación superior.

En cuanto a los resultados, el objetivo propuesto en la investigación se logró, toda vez que los estudiantes fueron participes de la integración de los dispositivos móviles. Asimismo, los estudiantes reflexionaron y trabajaron a fondo el problema de investigación desde la priorización de problemas (Pérez-Uribe, 2012). De acuerdo con los instrumentos para la recolección de la información, se concluye que el $63 \%$ de la muestra prefiere utilizar los dispositivos móviles, para actividades académicas, lo cual es un factor que favorece la inclusión del Aprendizaje Móvil (Estrada, 2014).

Respecto a la estrategia aquí propuesta se buscó en una herramienta gratuita para los estudiantes interesados en la delimitación de problemas de investigación. La cual se compone de contenidos digitales basado en el $m$-learning que los llevan a plantear sus proyectos de investigación.

\section{Referencias}

Barbolla, C., Benavente, N., López, T., Martín, C., Perlado, L., \& Serrano, C. (30 de 11 de 2010). Investigación etnográfica, métodos de investigación educativa en Ed. Especial. Recuperado el mayo de 2013, de http://www.uam.es/personal_pdi/stmaria/jmurillo/InvestigacionEE/Presentaciones/Curso_10/I_Etnografica_Trabajo.pdf

Barbolla, C., Benavente, N., López, T., Martín, C., Perlado, L., \& Serrano, C. (30 de noviembre, 2010). Investigación etnográfica, métodos de investigación educativa en Ed. Especial. Retrieved Mayo de 2013 from http://www.uam.es/personal_pdi/stmaria/jmurillo/InvestigacionEE/Presentaciones/Curso_10/I_Etnografica_Trabajo.pdf

Bautista, N. P. (2011). Proceso de la investigación cualitativa. epistemología, metodología yaplicaciones. Bogotá D.C: Manual Moderno. ISBN 9789589446409

Bolívar, W., Ochoa, M., \& Orrego, C. (2014). Capacitación B-learning, cambios en la práctica docente. Rev. Ciencia y Poder Aéreo, 9 (1), 167-181.

Bottorff, J. L; Boyle, J.S; Carey, Martha Ann, et al. (2003). Asuntos críticos en los métodos de investigación cualitativa (J. Morse, Ed.). Medellín, Antioquia: Edit. Universidad de Antioquia (1ra Edición). ISBN 9789586556569 
Consejo Nacional de Acreditación. (2010). Lineamientos para la acreditación institucional. Bogotá : CNA.

Escuela de Postgrados Fuerza Aérea Colombiana. (2012). Reglamento de Investigación. EPFAC: Departamento de Investigación.

Escuela de Postgrados Fuerza Aérea Colombiana. (2013). Documento Maestro Especialización en Gerencia de la Seguridad Operacional-SNIES 102769. Bogotá: EPFAC.

Estrada-Villa, E. J. (2014). Factores que contribuyen y dificultan el desarrollo de la enseñanza aprendizaje mediada por dispositivos móviles en Instituciones de Educación Superior en Colombia. Disponible en http://intellectum.unisabana.edu.co/ bitstream/handle/10818/11596/Erika\%20Juliana\%20Estrada\%20Villa\%20(tesis).pdf?sequence=1: Tesis de Maestría.

Estrada-Villa, E. J., Rodríguez Gallego, O. C., Gutiérrez Barbosa, P., \& Jaramillo Cortés, L. E. (2015). Hacía una metodología para la enseñanza del idioma inglés en la modalidad virtual para la Escuela de Postgrados de la Fuerza Aérea Colombiana. Revista de Tecnología Aeronáutica, 24, 23-29. Disponible en https://www.researchgate.net/publication/295547993_Looking_for_a_Virtual_English_teaching_system_for_the_Colombian_Air_Force_Graduate_School.

FAC. (2013). Directiva Permanente FAC No. 028. Bogotá: COFACJEMFA-JEA-DICTI-26.1 d.

Fernández Ordóñez, G. M. (2009). Generador e Interprete QR Code. Sevilla: Tesis de la Escuela Técnica Superior de Ingeniería Informática.

Luque Ordoñez, J. (2012). Códigos QR . ACTA - Autores científicotécnicos yacadémicos, 9-28.

Ministerio de Educación Nacional . (2010). Decreto 1295. Bogotá: Republica de Colombia

Orrego, C., \& Alba, M. (2013). Aprender haciendo en la virtualidad. Ciencia y Poder Aéreo, 8 (1), 108-115. DOl: http://dx.doi. org/10.18667/cienciaypoderaereo.14

Perez, J. F., \& Lagos, S. D. (2014). Diagnóstico para el uso de la realidad aumentada como recurso didáctico en UNAH-TEC Danlí. Herramient@s UNAHINNOV@ No.3, 21-27.

Pérez-Uribe, R. (2012). Priorización de problemas en ambientes de seguridad operacional. Ciencia y Poder Aéreo,7 (1), 8-19. doi:http://dx.doi.org/10.18667/cienciaypoderaereo.16

Tony Bates, A.W., (2001). Cómo gestionar el cambio tecnológico: estrategias para los responsables de centros universitarios. Barcelona: GEDISA. 